\title{
Research on the Three Famous Pattern Blocks in China
}

\author{
Ze-Yang PENG ${ }^{1, a}$ \\ ${ }^{1}$ College of Fashion, Wuhan Textile University, Wuhan, 430073, China \\ a754914234@qq.com,
}

Keywords: Women's pattern, Japan new cultural basic pattern, Donghua basic pattern, Rui-pu liu basic pattern, Structure, Comparison.

\begin{abstract}
This paper mainly for new Japanese cultural pattern, Donghua pattern and Rui Pu Liu of the third-generation pattern of the comparison and analysis of the research. Firstly, we study the human basis for the establishment of the pattern, including the neck, shoulders, chest, back, abdomen, upper part of the structure and to the structure design of control; then we analyze the formation of three kinds of patterns and specific analysis; after that we analyze comparing the three patterns research methods and the structural size. Summing up, Rui Pu Liu pattern uses less data, formula is simple and easy to operate, above the armhole depth, suitable for making a loose clothing or some conventional clothing and darts distribution is even and smoother. Donghua pattern and the Japanese pattern is suitable for application in a tight-fitting clothing structure design. Japanese pattern is bodice pattern and is divided into a number of pieces, more suitable for a tight dress, small suit and fashion design. After all the analysis and balanced check, we found the Donghua pattern to the best, followed in rank by Japanese pattern and Rui Pu Liu pattern at the last.
\end{abstract}

\section{Introduction}

Basic block is the starting of all the pattern making process, therefore, an accurate pattern drafting method can help create perfect basic blocks that ensure the apparel fit and balance. There are many methods used to making pattern which are famous for their accuracy and fit. However, the Chinese garment industry seem loath to adopt these methods as the apparel balance of these methods are not being used for Chinese body type. This research is aimed at looking for the perfect fitting pattern method for Chinese body type through the comparison of 3 major patternmaking methods in china with under the theory base of anthropometry. Initial body measurement database, patternmaking method details and garment ease database, are first built for pattern making experiment. The measurement of the standard dummy together with the body sizes in GB form the anthropometry database. The making method database is created with a study of each pattern making method. The ease distribution of each basic block is calculated, as to form the apparel ease database. And the initial body measurement database for the pattern making and sample making are built. The experiment is based on the method of close-fitting bodice block of three kind of pattern through pattern making and sample making. Using 2D and 3D analysis, a comparison analysis is made on the aspects of garment balance and garment fit. The 2D analysis is made from the method of image combination. A prediction is made on the position and balance of apparels discussions and analyses.

\section{The Formation and Characteristics of Three Kind of Basic Patterns}

\section{The Formation and Characteristics of Donghua}

In 1988, Donghua University started researching the Chinese clothing pattern. After many years of efforts, they eventually made this basic pattern. In 2006, Zhang Wenbin's book, "Design of the clothing" which is about the definition of Donghua basic pattern, explained the regression equation relationship between body parts, the height and bust girth. Donghua basic pattern measured a large number of women's measurements. What's more, using the female body of the measured data of the average to established the standard dummy, and then in the standard dummy by Donghua pattern of the original method of making the cloth to simplified the relationship between body details, height, 
and the regression equation. Finally, drafting Donghua basic pattern process as an original method developed for Chinese women's box pattern block. [1] Picture 1 show us the character of Donghua basic pattern, first the formula from SNP to BL is $0.1 \mathrm{~h}+8.7$ which is connected to height; secondly, front-shoulder sloping is 24 degrees and the back-shoulder sloping is about 15 degrees. Eventually, we can see the waist line is parallel with the floor, which means this method's benefit without bust darts transfer into the waist line.

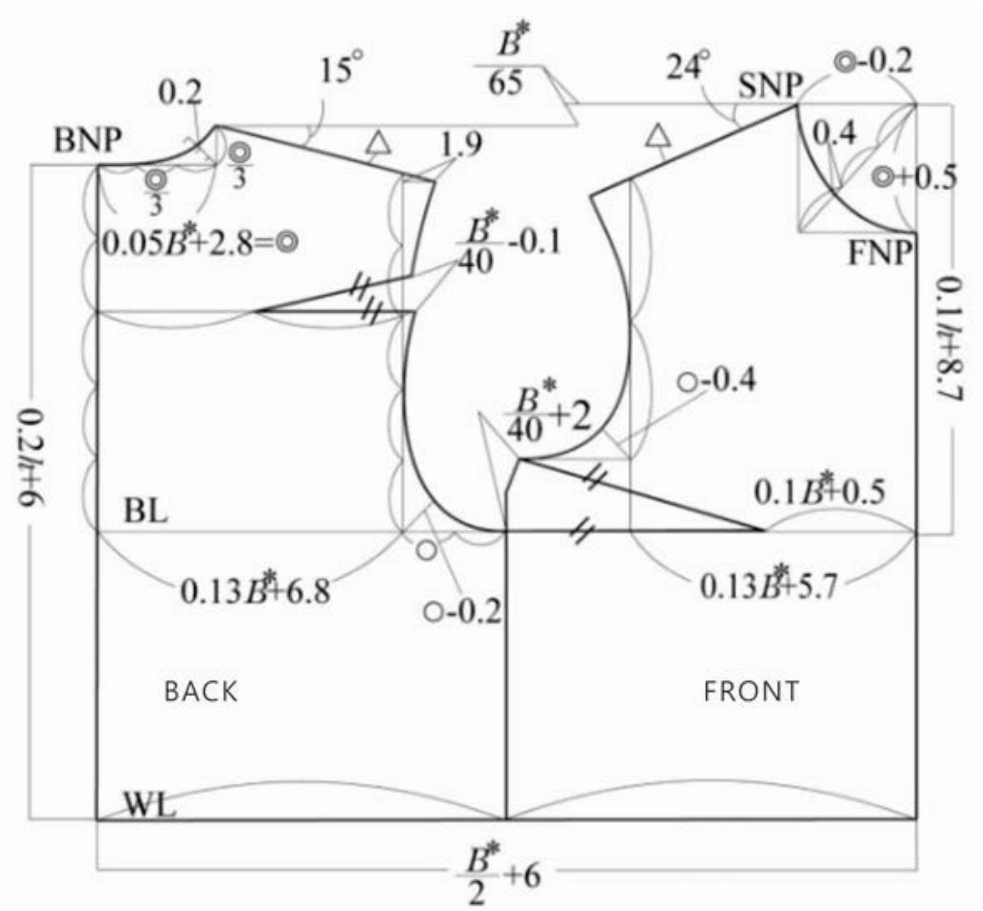

Fig. 1 Donghua basic pattern block

\section{The Formation and Characteristics of Rui-Pu Liubasic Pattern}

Liu Rui-Pu's basic pattern came from the book "Design principles of clothing structure” in 1990. [2] It adopted Japan's size in his own book with international standards and using back length and bust girth as initial data to calculate another parts measurement. Using this method to make pattern block, we call it Rui-Pu Liu basic pattern. From picture 2, we come to know that this kind of pattern maintains two special characters. Firstly, changing the armhole depth formula from BG/6+7 to BG/6+6.5 can make the tightness in the bust and armhole improved. Secondly, this method discharges the back width with $0.5 \mathrm{~cm}$ so the difference between front width and back width can be reduced. When the lowest armhole point up is $0.2 \mathrm{~cm}$, we can see that the armhole line become smoother than before. Smooth armhole ling reduces arm flexion disorder and gives comfort to the back of the shape. 


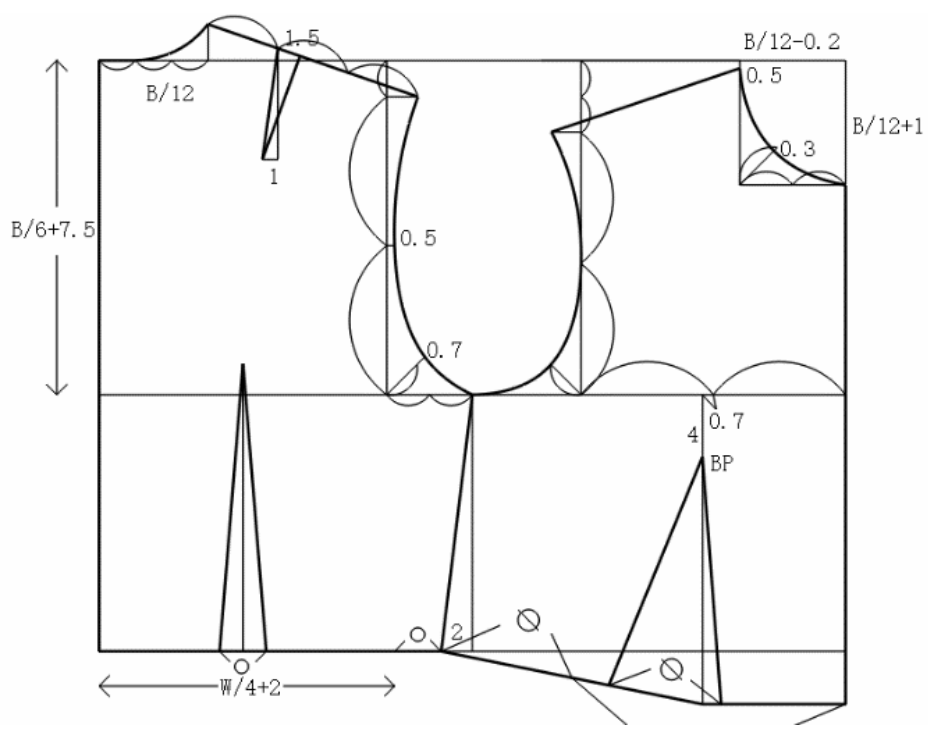

Fig. 2 Rui-Pu Liu basic pattern block

\section{The Formation and Characteristics of Japanese Basic Pattern}

The Japanese pattern has gone through eight changes from its inception to the present [3]. From picture 3, we can know that their change is in-line with terms of the human body, costumes and other human activities, mainly in the following areas. Firstly, the shoulder sloping has changed which is maximum in the first generation of pattern shoulder sloping with an average of 25 degrees that is suitable for oblique shoulder of the human body. From the first-generation pattern to the eighth-generation pattern, the angle of the shoulder line of 25 degrees is gradually reduced to 22 degrees which reflects the Japanese pattern of people-oriented mapping concept. Secondly, the decline of the front center line reflects that the Japanese women's chest is constantly changing at a rate of $0.2 \mathrm{~cm}$ per average decline. Finally, the ratio of chest width to back width is constantly adjusted, reflecting the Japanese pattern in the body to meet the activities of the body with a good comfort.

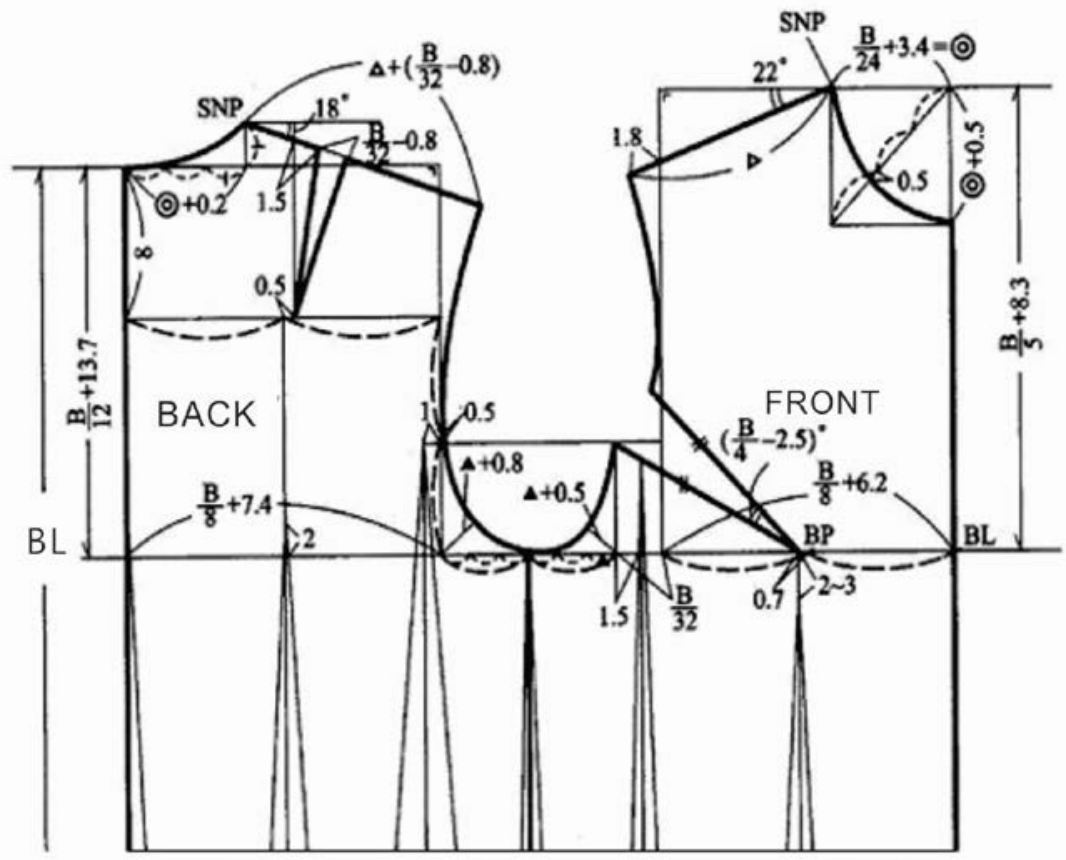

Fig. 3 Japanese basic pattern block

In summary, the drawing process description of the three patterns is drawn into table 1 . As shown 
in the following table, we can see that three kinds of patterns cartography when using the formula B / $2+6 \mathrm{~cm}$, one of the $6 \mathrm{~cm}$ for the chest can be understood as chest loose volume. But for the entire upper body, the pattern forms the necessary size. In the human body, this pattern cannot be obtained through a two-dimensional measurement but through the upper part of the upper body cross-section coincidence map to obtain the smallest outer circumference after the calculation.

Table 1 Comparison of three patterns

\begin{tabular}{|c|l|l|l|l|}
\hline Nu & program & Japan pattern & Donghua pattern & Ruipu Liu pattern \\
\hline 1 & back length & 38 & $0.2 \mathrm{~h}+6=38$ & 38 \\
\hline 2 & front center - back center & $\mathrm{B} / 2+6=48$ & $\mathrm{~B} / 2+6=48$ & $\mathrm{~B} / 2+6=48$ \\
\hline 3 & $\mathrm{BNP}-\mathrm{BL}$ & $\mathrm{B} / 12+13.7=20.7$ & $0.1 \mathrm{~h}+8.7=21.1$ & $\mathrm{~B} / 6+7.5=21.5$ \\
\hline 4 & back width & $\mathrm{B} / 8+7.4=17.9$ & $\mathrm{~B} / 8+6.8=17.3$ & $\mathrm{~B} / 6+5=19$ \\
\hline 5 & SNP-BL & $\mathrm{B} / 5+8.3=25.1$ & $0.1 \mathrm{~h}+8.7=24.7$ & $\mathrm{~B} / 6+7=21$ \\
\hline 6 & chest width & $\mathrm{B} / 8+6.2=16.7$ & $\mathrm{~B} / 8+5.7=16.2$ & $\mathrm{~B} / 6+3=17$ \\
\hline 7 & front neck width & $\mathrm{B} / 24+3.4=6.9$ & $\mathrm{O}-0.2=6.8$ & $\mathrm{O}-0.2=6.8$ \\
\hline 8 & front neck deep & $\mathrm{O}+0.5=7.4$ & $\mathrm{O}+0.5=7.5$ & $\mathrm{O}+1=8$ \\
\hline 9 & back neck width & $\mathrm{O}+0.2=7.1$ & $\mathrm{~B} / 20+2.8=7$ & $\mathrm{~B} / 12=7$ \\
\hline 10 & back neck deep & $(\mathrm{O}+0.2) / 3=2.4$ & $\mathrm{O} / 3=2.3$ & $(\mathrm{O} / 3=2.3$ \\
\hline 11 & bust dart & $\mathrm{B} / 12+3.3=3.7$ & $\mathrm{~B} / 40+2=4.1$ & 1 \\
\hline 12 & shoulder dart & $\mathrm{B} / 32-0.8=1.8$ & $\mathrm{~B} / 40-0.1=2$ & 1.5 \\
\hline 13 & front shoulder sloping & $22^{\circ}$ & $24^{\circ}$ & $19^{\circ}$ \\
\hline 14 & front shoulder sloping & $18^{\circ}$ & $15^{\circ}$ & $19^{\circ}$ \\
\hline & & & & \\
\hline
\end{tabular}

\section{Three Kind of Pattern Two-Dimensional and Three-Dimensional Analysis}

From the three-dimensional perspective of the three types of pattern analysis, Donghua pattern for the box structure, this kind pattern block balance is better than the Japanese pattern and the Ruipu Liu pattern. Donghua pattern and the Japanese pattern of the chest position are in the armhole which led to the above two patterns of the clothes in the same level and it gives a good sense of vision as well as the clothes don't have too much folds and upturned. But Rui-Pu Liu pattern of the bust dart has been transferred to the clothing position which led to this pattern of the cloak and the waist line is not parallel with the floor, although the overall pattern is balanced but a little adjustment is needed and as there is no excessive fold but it needs to be adjusted before use. From the side to see the three armhole arc of the patterns, they are relatively rounded without a big turning point. Three pattern armhole depth are appropriate from the above table; Rui-Pu Liu pattern's armhole lines being the lowest, Donghua pattern following and the Japanese pattern being the highest, indicating that the pattern of Rui-Pu Liu is fit, Donghua pattern is fitter while the Japanese pattern the fittest. 


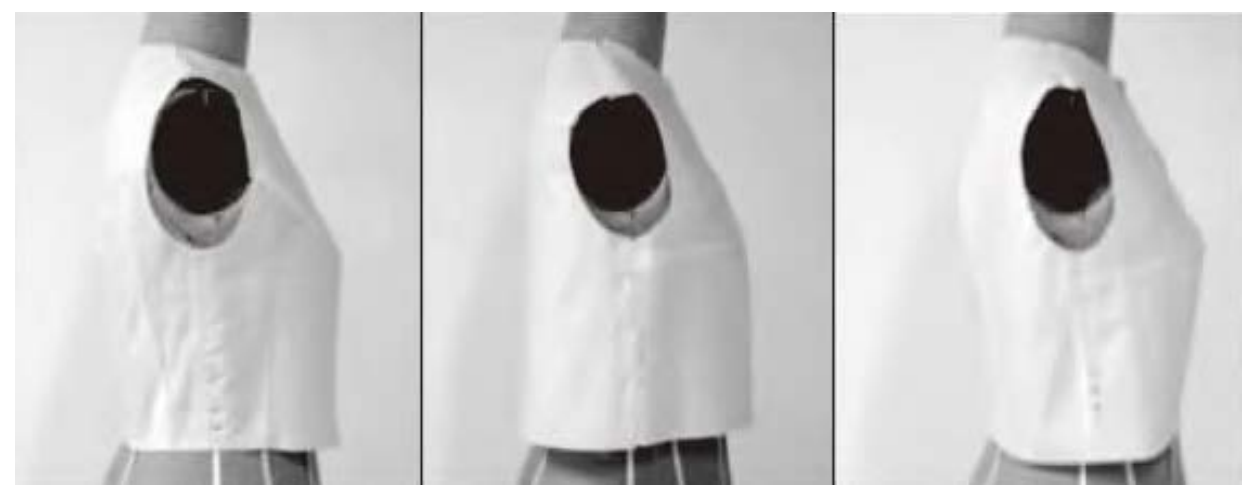

Fig. 4 Donghua basic pattern block, Rui-Pu Liu basic pattern block, Japanese basic pattern block

After closing the armhole points of all the three types of patterns, the two-dimensional sketches of the three patterns were analyzed. Firstly, in the three kinds of patterns, the bust volume and size are the same, but after overlapping the three patterns, we can find that the front center line and the centerline is not on the same line, which means that even if we use the same set of data while using different regression equations, the results are inconsistent. Secondly, we can find that these pattern blocks' waist lines don't lie in the same line so we think that maybe this is caused by the back length. Donghua pattern using the $0.2 \mathrm{~h}+6$ as it, but Rui-Pu Liu pattern and Japanese pattern using the same value $38 \mathrm{~cm}$. Finally, from the bust point we can find that their air gap is different. Rui-Pu Liu basic pattern front center line is far away from other two patterns so we believe Rui-Pu Liu basic pattern have the biggest air gap.

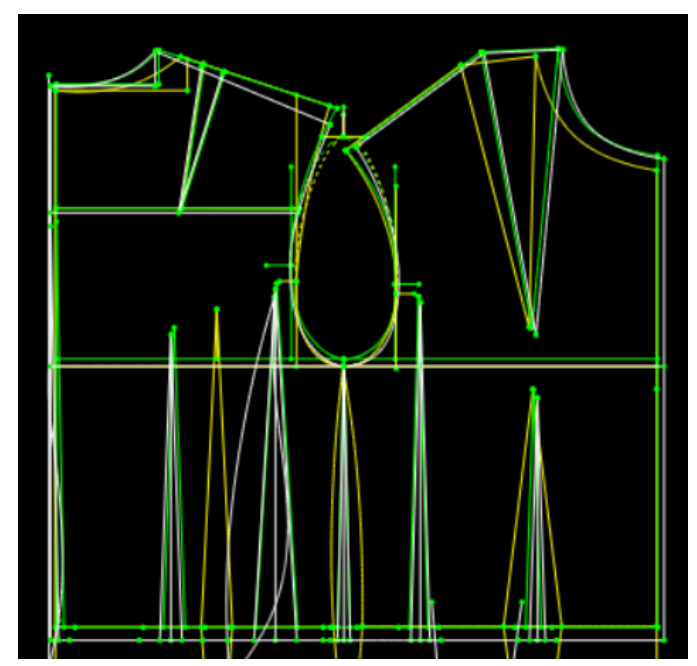

Fig.5 Three kinds of patterns overlap picture

\section{The Balance Analysis of Three Kind Patterns}

Garment balance includes three kinds which are the front balance, the back balance and the side balance. According to pictures 1-5 we believe that the effects of balance maintains the back of the human body and the difference between the length of clothing, the difference between the length of the arm and the arm of the human body. The difference between the length of the garment front and the body front. As the picture 6 . 

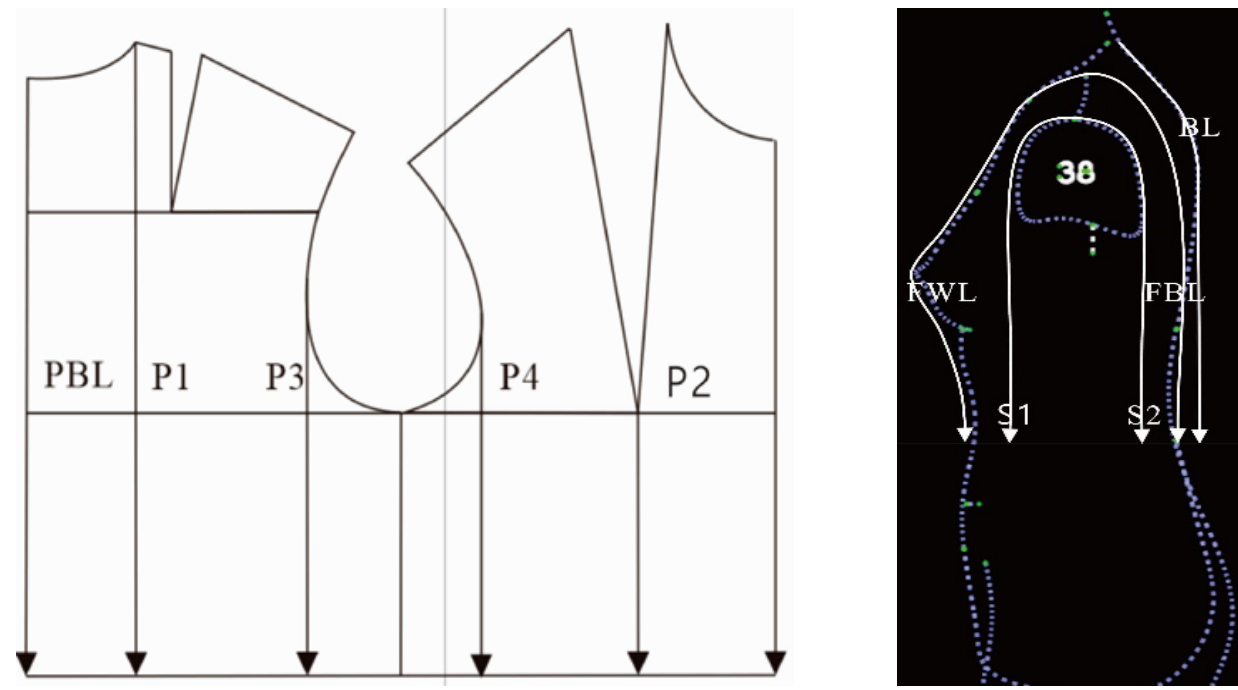

Fig.6 garment and body balance picture

As is shown above, firstly, we can name the back balance as B1 and the formula is B1=PBL-BL. Secondly, the front balance which we call B2 and we think B2 $=\{(\mathrm{P} 1+\mathrm{P} 2)-(\mathrm{FWL}+\mathrm{FBL})\} / 2$ is good. Thirdly, we use $\mathrm{B} 3$ to represent the side balance and the formula is $\mathrm{B} 3=\{(\mathrm{P} 3+\mathrm{P} 4)-(\mathrm{S} 1+\mathrm{S} 2)\} / 2$. When $\mathrm{B} 1=\mathrm{B} 2=\mathrm{B} 3=0$ we can believe that this pattern is balanced. Because of the fabric thickness and sewing technology, we think that $\mathrm{B} 1=\mathrm{B} 2=\mathrm{B} 3=0$ is impossible so we assume $\Delta \mathrm{B}$ is the maximum difference between B1, B2 and B3 and the formula is $\Delta \mathrm{B}=\max \{|\mathrm{B} 1-\mathrm{B} 2|,|\mathrm{B} 2-\mathrm{B} 3|,|\mathrm{B} 1-\mathrm{B} 3|\}$. When $0<\Delta B<0.3$ we also think there are balanced. When we use this formula to calculate these patterns, we will get the values in table 2. In this table, A means Donghua basic pattern block, B means Rui-Pu Liu basic pattern block and C means Japanese basic pattern block.

Table 2 Three kind patterns balance detection

\begin{tabular}{|l|l|l|l|l|l|l|l|l|}
\hline & $\mathbf{P}_{\text {BL }}$ & $\mathbf{F W L + F B L}$ & $\mathbf{P}_{\mathbf{3}}+\mathbf{P}_{\mathbf{4}}$ & $\mathbf{B 1}$ & $\mathbf{B} \mathbf{2}$ & $\mathbf{B} 3$ & $\mathbf{\Delta} \mathbf{B}$ & Balance detection \\
\hline $\mathbf{A}$ & 38 & 81.5 & 70.6 & 0 & 0 & 0.05 & 0.05 & Balance \\
\hline $\mathbf{B}$ & 38 & 82.8 & 71.8 & 0.4 & 0.65 & 0.65 & 0.25 & Balance \\
\hline $\mathbf{C}$ & 38 & 81.6 & 70.4 & 0 & 0.05 & -0.05 & 0.1 & Balance \\
\hline
\end{tabular}

As shown in the above table, according to the principle of balance to calculate it we can find three kinds of patterns have reached a balanced state conclusion and the balance $\mathrm{A}$ is best and the $\mathrm{B}$ is good and $\mathrm{C}$ is better.

\section{Conclusion}

(1) Three patterns, the Japanese cultural prototype bust and shoulder dart values are greater, but waist dart distribution from the front and back has no extra folds, highlighting the body's natural curves. Chest width and back width is moderate and the front shoulder pattern fits on the body, but the shoulder is not fully fit, while armhole better shape before the lumbar section of the waist line and are substantially flush at waist fit body garment pattern but the formula of this pattern is not easy to use.

(2) Donghua pattern in 3 kinds of pattern armhole shape most comply with the principle of arm forward movement, shoulders, chest and shoulder darts are moderate, but box style pattern is suitable for making class clothing, suitable for clothing. Back piece slightly low not very beautiful when clothes are static. Calculation formula of the denominator for double-digit is slightly complicated while usage. In accordance with the shoulder blade, the dart is located at the armhole, which are different from the other two patterns.

(3) Rui-Pu Liu pattern uses less data and the formula is simple and easy to operate. When the armhole depth has comfort, loose clothing production will be better. Donghua pattern and Japanese pattern are suitable for making tight clothing, especially the cultural pattern uses a tight design, when 
making a tight suit will be more solid.

(4) In general, these three patterns are the use of bust as the standard for calculating the size of other parts. They are using the back length as the basis for looking for waistline. Their results are based on a large number of human data collection after the calculation, so more scientific. The above is the result of the study of the author hope to provide reference for other scholars.

\section{References}

[1] Zhang, Wei-Yuan Zhang. Chinese clothing prototype - Donghua prototype structure design principle and technology [J]. Journal of Donghua University: Natural science edition, 2002, 28 (3) : 44-50.

[2] Liu RuiPu. Design principles and application of garment design [M]. Beijing: China textile publishing house, 2008.09.01

[3] SanJi Zhi-Zi. Theory of clothing modeling [M]. First edition. Beijing: China textile publishing house, 2008.06 